\title{
Review on Personality Types and Learning Styles in Team-based Learning for Information Systems Students
}

\author{
Muhammad Zul Aiman Zulkifli , K.S. Savita ${ }^{2}$, Noreen Izza Arshad ${ }^{3}$ \\ Computer and Information Science Department \\ Universiti Teknologi PETRONAS \\ Perak, Malaysia
}

\begin{abstract}
Team-based learning (TBL) has become a preferable method in learning approach at higher educational level. There are a lot of articles that discussed on the benefits and process of implementation of team-based learning but lack of studies that focus on the composition of members in teambased learning and effects of personality types and learning styles towards it. This article set out to analyze the existing literatures on team-based learning implementation at undergraduate and how personality types and learning styles affected the learning process plus exploring these topics in information systems field. Guided by Okoli systematic review method, a systematic review from Scopus, Web of Sciences and Association of Information Systems (AIS) databases has been conducted. Results shows that TBL received positive feedback from the scholars but only have issues on the implementation process consist of the usage of student's personality and learning styles, role of team members, TBL management in classroom, TBL is not "fit for all" and current studies about TBL. The usage of personality and learning style instruments is one of the suggested ways to improve it but there are no details guidelines available yet on how to use it. There is lack of studies about team-based learning in information systems field.
\end{abstract}

Keywords-Team-based learning; personality type; learning style; undergraduate students

\section{INTRODUCTION}

Team-based learning is a highly designed teaching and learning strategy that maximises student preparation and participation, giving students' responsibility for their own learning before and during class session. Students spend time in class solving authentic problems in essentially self-managed, high-performing, permanent teams [1]. This approach requires students to apply learned knowledge to solve significant, authentic and complex scenarios individually and within a team [2]. Essential elements in TBL, consist of groups, accountability, feedback and assignment design [3]. The explanation for the elements are (1) groups - groups must be properly formed and managed, (2) accountability - students must be accountable for the quality of their individual and group work, (3) feedback - students must receive frequent and timely feedback, and (4) assignment design - group assignments must promote both learning and team development.
Team-based learning play a major role in promoting team learning among students. This can be seen with team learning become a demand within organization as the increasing global competition, consolidation, and innovation. Furthermore, team members affects the degree of the effectiveness of the team as it exhibits the expertise diversity and collective identification towards group performance [4]. In addition, working in group become more beneficial when the tasks and project requires a larger skill and commitment from the member to be completed especially in project involving completed and participation of multiple field [5].

This paper attempt to explore and understand the trends of the research's topics and identify the relationship between personality types and learning styles of undergraduate students within the process of team-based learning. Additionally, this study is vital because team learning becoming a demand within an organization as they requires the use of teams at all hierarchical level [6]. Working in team can be train before the students graduate into working field and higher education institution is one of the suitable medium to nurture it. Therefore, details on where past literatures has so far focused provide the opportunity in understanding on where the emphasis is and where the attention need to be placed. To construct a relevant systematic review, the current article is guided by the main research question - How learning styles and personality of undergraduate students affect team-based learning process? Focus is given on student's personality and learning style as these components may affects the group effectiveness when conducting team-based learning [7], [8]. Thus, this study attempts to investigate the effects of learning styles and personality types on team-based learning among undergraduate students across all fields with emphasize on information systems field.

This section explains the purpose of conducting systematic literature review while the second section details out the methodology process. The last section discussed on the current trends around TBL and its integration between personality and learning styles of learners.

\section{METHODOLOGY}

The author conducted a systematic review by following guideline by Okoli systematic review protocol [9]. A systematic review is a review of a clearly formulated question that uses 
systematic and explicit methods to identify, select, and critically appraise relevant research, and to collect and analyse data from the studies that are included in the review [9][10]. It follows a rigorous and scrupulous procedure to search and select the sample studies for coding and analysis. It is a methodical and meticulous process of collecting and collating the published empirical studies of acceptable quality with systematic criteria for selection to reduce researcher bias and provide transparency to the process.

\section{A. Systematic Review Protocol}

Protocol can be described as a document written before the start of a systematic review describing the rationale and intended purpose of the review, and the planned methodological and analytical approach [10]. According to Okoli review protocol [9], there are eight steps need to be followed when conducting systematic review. These steps are been followed in this paper's review protocol and the explanation are detailed in the following statements.

First step is to identify the purpose of this paper. Align with its objective, this paper reviews the current research on personality types and learning styles of undergraduate student within team-based learning implementation. The systematic review is focusing on domain review where it will highlight the empirical findings of the reviewed papers. All field of studies are included, and more focus is been given in information systems. The second step is drafting the searching protocol. Major search term is been extracted from the research question which are learning styles, personality types and team-based learning. Then, the author identifies the relevant terms, synonyms and alternative spelling that are used in the published literatures as shown in Table I.

Third, the steps continued with developing search strings to find the papers in the databases. The search strings are developed as stated below:

TITLE-ABS-KEY(("team-based learning" OR "team based learning" OR "team learning" OR "group-based learning" OR "group based learning" OR "group learning") AND (("learning style*" OR "learning strateg*" OR "learning approach" OR "learning method") OR ("personalit* type*" OR "personality")) AND ("undergraduate*" OR "under graduate*" OR "higher education" OR "university" OR "degree")).

These search strings will be used in the selected databases to find papers. The selected databases are Scopus, Web of Sciences and Association of Information Systems (AIS). Scopus and Web of Sciences are selected because it the database contain only high-indexed journal while AIS is selected as the focus is to explore more in information systems (IS) thus giving opportunity to the author to find the results of the articles in IS domain.

The fourth step is screening for inclusion. After getting the results from the databases, the articles will be filtered based on the criteria imposed by authors. The screening is important to make sure the papers get is within the topic's domain and sufficient while at the same time the excluded articles do not affect the quality content of this systematic review paper. The articles are selected for review if: (1) Using any of research keyword. (2) Using any of the research questions or attempt to describe its nature. (3) Published in or submitted to conferences or journals. (4) Written in English. (5) Published within 2015 to March 2019. (6) Related to the topics such as active learning and flipped classroom. On the contrary, the publication will exclude if they were: (1) Papers with no empirical findings (e.g. review paper). (2) Papers discussing completely different area from research topics. (3) Masters and $\mathrm{PhD}$ studies which are not published in any referred conferences or journals. (4) Informal literature survey (no defined search questions, no search process, no defined data extraction, or data analysis process). (5) Papers with no answer relevant to the research questions. The process of selecting the paper for review is shown at Fig. 1.

Fifth, after filtered all these articles, data extraction process is conducted. During this phase, author will review each article with its objective to answer these following questions: (1) How personality types and learning styles where been integrate in the study? (2) How the study was conducted? What are the results of the study? (3) What are the future recommendations from the study? (4) What are the limitations of the study? The results from data extraction phase are explained in results section.

Sixth, the next process is quality appraisal. After extracting the needed information from all the papers, the papers are going through quality appraisal process to prioritize the papers according to their quality and to exclude certain papers deemed to not useful due to inferior methodological quality. The components that are highlighted in this process are stated as follows: (1) What claims the papers make? (2) What are the evidences they provide to support the claims? (3) Are the evidences are warranted?

TABLE I. KEYWORDS SYNONYMS FOR SEARCH STRING

\begin{tabular}{|c|c|c|}
\hline Keywords & \multicolumn{2}{|l|}{ Synonym } \\
\hline $\begin{array}{l}\text { Team-based } \\
\text { learning }\end{array}$ & \multicolumn{2}{|c|}{$\begin{array}{l}\text { team based learning, team learning, group- based } \\
\text { learning, group-based learning, group learning }\end{array}$} \\
\hline Personality type & \multicolumn{2}{|l|}{ personality } \\
\hline Learning style & \multicolumn{2}{|c|}{$\begin{array}{l}\text { learning approach, learning strategy, learning } \\
\text { technique, study style, study approach, study strategy }\end{array}$} \\
\hline Undergraduate & \multicolumn{2}{|c|}{ undergraduate, higher education, university, degree } \\
\hline Identification & $\begin{array}{c}\text { SCOPUS }=291 \\
\text { WOS }=124 \\
\text { AIS }=497\end{array}$ & \multirow{2}{*}{$\begin{array}{l}\text { Excluded paper that are } \\
\text { note related at the online } \\
\text { database site }\end{array}$} \\
\hline \multirow{3}{*}{$\begin{array}{l}\text { Eligibility / } \\
\text { Screening } \\
\text { for inclusion }\end{array}$} & $\begin{array}{c}\text { SCOPUS }=104 \\
\text { WOS }=137 \\
\text { AIS }=71\end{array}$ & \\
\hline & $\begin{array}{c}\text { SCOPUS }=34 \\
\text { WOS }=33 \\
\text { AIS }=17\end{array}$ & $\begin{array}{l}\text { Excluded paper that are } \\
\text { note related after } \\
\text { reviewing the abstract } \\
\text { and title }\end{array}$ \\
\hline & & \multirow{2}{*}{$\begin{array}{l}\text { Excluded paper that are } \\
\text { note related after full } \\
\text { reading, remove } \\
\text { redundant articles, } \\
\text { remove articles that faced } \\
\text { difficulties to retrieve full } \\
\text { version }\end{array}$} \\
\hline $\begin{array}{l}\text { Finalized } \\
\text { articles for } \\
\text { analysis }\end{array}$ & $\begin{array}{c}\text { SCOPUS }=9 \\
\text { WOS }=8 \\
\text { AIS }=0\end{array}$ & \\
\hline
\end{tabular}

Fig. 1. Searching Protocol used to Select Articles. 
Seventh, after conducting quality appraisal process, the data are synthesized using thematic analysis. The findings are been explained in the results section. The last process is to write the review which is the content of this paper itself.

\section{RESULTS AND DISCUSSION}

Based on the selection process stated in methodology section, 17 articles are selected for this review as shown at Table II. From these articles, the content of the articles had been analyzed and the results are presented as follows.

TABLE II. SELECTED ARTICLES FOR REVIEW

\begin{tabular}{|l|l|}
\hline Watkins et al. (2018)[11] & $\begin{array}{l}\text { Salimath, Vijaylakshmi, \& Shettar (2018) } \\
{[12]}\end{array}$ \\
\hline Jeno et al. (2017) [13] & Rezaee, Moadeb, \& Shokrpour (2015) [14] \\
\hline Nicole \& Larson (2016) [15] & Kim, Song, Lindquist, \& Kang (2016) [16] \\
\hline Hettler (2015) [17] & Frame et al. (2015) [18] \\
\hline Iramaneerat \& Ba (2017) [19] & $\begin{array}{l}\text { Miller, Khalil, Iskaros, \& Van Amburgh } \\
\text { (2017) [20] }\end{array}$ \\
\hline Stepanova (2017) [21] & Obad et al. (2019) [22] \\
\hline $\begin{array}{l}\text { Behling, Murphy, \& Lopez } \\
\text { (2017) [23] }\end{array}$ & Ismail (2016) [24] \\
\hline Frame et al. (2016) [25] & $\begin{array}{l}\text { Kenny, Mclaren, Blissenden, } \\
\text { \& Villios (2015) [26] }\end{array}$ \\
\hline $\begin{array}{l}\text { Greetham \& Ippolito (2018) } \\
\text { [27] }\end{array}$ & \\
\hline
\end{tabular}

\section{A. Student's Personality and Learning Style in TBL}

From this search, there are two studies that mentioned the involvement of personality or learning style instrument in their researches: Myers-Briggs Type Indicator (MBTI) [18] and VARK [24]. For MBTI, study shows that this instrument help in creating the team for TBL. They suggest that the personality can become a key role in team success as student believe diversity in team contribute for better functioning group. Knowing team members personalities allows instructor to identify each individual their strengths and weaknesses and make them to be put in the right team that may give them opportunity to display their capabilities to others. At the same time, knowing personalities allows the members to distribute the tasks based on each capability.

Looking at Ismail [24], although the study does not mention VARK, they indirectly highlighted the component of VARK which are visual, audio, reading and kinesthetic and how these can help their students to incorporate their learning style in classroom. Understanding their learning styles help the teams to recognize each other capabilities and allows the distribution to be handle effectively. Other studies also mentioned that understanding student learning style will help to avoid clashing within the team. Student that know their preferences can use it to facilitate their learning for maximum results. From this, personality and learning styles will tackle the same issues in understanding the student's capabilities especially when working in team. Thus, using both personality and learning style instrument will add more value in getting the insights of each member's capabilities. Although the studies highlight the importance of personality and learning style, the are no details model available on how to form the group using these instruments. Further researches are needed for verification on the details of combination and synchronization between personality and learning style components.

\section{B. Team Members Play Role in Effective TBL}

In TBL, the composition of team members in a group plays major role in determining the successfulness of the learning method. Greetham [27] expressed that TBL help in maximizing learning outcome with better team dynamics, applying the knowledge into more technical tasks and enhance the process of subject mastery. In getting the team dynamics, the formation of the members is crucial. Stepanova [21] expressed that instructor should look on the formation of the team to make sure the team are productive for TBL successfulness. As TBL is known as learner centric approach, getting a right member for team is the first crucial step. There are few suggestions arise regarding the formation of the team. Frame [18] found that group formed with heterogonous members are more successful compare to homogenous members. The members are formed based on MBTI and the success are based on the student's survey. They also emphasized on understanding the student's different personality and their learning style approaches and use it to form the group.

\section{Managing TBL in Classroom}

One of the uniqueness of TBL is its systematic approach compromise of preparation before class, readiness assurance test, application exercises and getting feedback [1]. This approach also means that managing TBL is important because several processes need to be done within limited time. Ismail [24] noted that time management must be properly restructured in TBL in ensuring the learning is time efficient and productive. Supporting this, Watkins [11] also listed time pressures as the challenges face when implementing TBL. They expressed that students faced the pressure of time when they have poor time management, multiple tasks and lack of commitment by other members when conducting the project. Thus, time allocation become an important factor in in handling TBL classroom because every process of TBL need to be executed to get the desired results. Plus, getting students opinions on time allocation of TBL in classroom also vital to know either TBL help them or make them feel overwhelm. These issues need to be highlighted as it happens commonly not only when using TBL but also in other active learning method and traditional classroom session.

\section{TBL is not 'Fit for All'}

Using TBL as the teaching strategy in the classroom does get variety of results from all type of students. Miller [20] and Behling [23] highlight that TBL are more appreciated by senior and older students compare to young and junior students. Senior students seem to more appreciated TBL method due to their understanding of past knowledge, experience in the real-life application and familiarity with peers. Meanwhile, Salimath [12] found that TBL is more suited in course that emphasizes in application in real world by performing course projects while non-TBL method is more suitable for students whose individual learning path are innovative and exposed to different learning environment. From this, it can be found that TBL is more suited to the courses that involve practicality and application towards 
industry and society while non-TBL session is more suited towards new students that still not familiar with higher education learning environment and theoretical courses.

Kim [16] emphasized that TBL cannot be seen as the panacea that suit all students. He pressed that as the teaching method, TBL should be evaluated from other various factors and more researches should be conducted pertaining the other factor as that may affects the TBL itself. Further researches are needed on TBL implementation in various field of studies and various educational level to know each field compatibility in using TBL method itself.

\section{E. Currents Studies on TBL}

From these articles, the distribution of the field of study the researches are conducted as follows: 10 medical field, 2 from engineering, 1 from computer sciences, 1 from social science, 1 from tax and accounting, 1 from business English and 1 from economics. This data shows that most of TBL researches are conducted around medical field. Meanwhile, there are study that came from other fields. This highlight that there is opportunity to conduct researches on TBL for others field as current researches shows that TBL may be fit in all field of studies, but further verification is needed.

For research designs, 12 studies are using quantitative methods, 2 studies using qualitative, 1 study using mixed methods and 2 studies are not clearly stated their research designs. Paper that using qualitative are mostly emphasizing on getting the effectiveness of TBL using the student's marks and feedbacks forms. For studies that using qualitative [11], [26], their focus more on understanding on how TBL works on their field as not much paper can be found within their related field. Study that use mixed method [21] doing both discovering and verification of TBL implementation of TBL in the classroom. For future studies, using qualitative method is preferably when researching TBL in new field of study such as information systems, information technology and other fields that still not familiar yet with TBL implementation. Using quantitative is recommended when doing verification and enhancement of TBL in already familiar field.

Looking at the information systems, this field is not yet applying team-based learning in the teaching class. This may due to the nature of the field that related with information technology (IT) and computer sciences that focus on technical and programming knowledge. Although this is true, information systems field is more towards bridging the technical and IT related entities with businesses application [28]. Thus, capability of information systems graduates to work in team is inevitable, making team-based learning method as a suitable approach in enhancing students' capabilities.

Nevertheless, more studies need to be done in measuring the successfulness of this approach in this field.

\section{CONCLUSION}

Using the approach of systematic review, the author managed to conduct a literature study on team-based learning and examining its relationship with student's personality and learning styles. Relevant literatures have been chosen for reviews and several aspects have been identified that directly involved in this topic. Overall, team-based learning (TBL) shows positive outcome in enhancing students learning outcome in the higher education. Most studies agree that TBL is a strategic learning approach that give benefits in enhancing students learning capabilities and working in the team. However, there still arising the issues are on the implementation of TBL itself. Here, the highlighted issues are on the usage of student's personality and learning styles, role of team members, TBL management in classroom, TBL is not "fit for all" and current studies about TBL.

Notably, there are lack of studies regarding these topics on information systems. It is clearly stated that TBL enhance the team learning among the students and information systems field also required the involvement of team either in learning or projects. Thus, it opens the opportunity for this field to explore this topic as different fields give different views and insights. From this study, there are several recommendation for future studies which are: (1) how to form the right group with the right mixture members to ensure the success of TBL implementation, (2) how to manage time effectively in TBL, (3) how other fields been affected when using TBL in their courses, (4) how far TBL can be implemented in information system field?

\section{REFERENCES}

[1] L. K. Michaelsen, M. Sweet, and B. C. Kelley, "Team-Based Learning," in New Directions for Teaching and Learning, no. 128, Wiley Online Library, 2011, pp. 35-41.

[2] J. Currey, S. K. Sprogis, G. Burdeu, J. Considine, J. A. Allen, and E. Oldland, "Students perceive Team-Based Learning facilitates development of graduate learning outcomes and professional skills,"J. Teach. Learn. Grad. Employab., vol. 9, no. 1, pp. 93-113, 2018.

[3] L. K. Michaelsen, M. Sweet, and D. Parmalee, "The essential elements of team-based learning,” in New Directions for Teaching \& Learning, vol. 2008, no. 116, 2008, pp. 7-27.

[4] S. Kozlowski and B. Bell, "Work groups and teams in organizations: Review update,” Handb. Psychol., vol. 12, pp. 412-469, 2013.

[5] S. Lavy, "Who benefits from group work in higher education? An attachment theory perspective," High. Educ., vol. 73, no. 2, pp. 175187, 2016.

[6] J. Mesmer-Magnus, A. A. Niler, G. Plummer, L. E. Larson, and L. A. DeChurch, "The cognitive underpinnings of effective teamwork: A Meta-Analysis,” Career Dev. Int., vol. 22, no. 5, pp. 507-519, 2017.

[7] T. F. Hawk and A. J. Shah, "Using Learning Style Instruments to Enhance Student Learning,” Decis. Sci. J. Innov. Educ., vol. 5, no. 1, pp. 1-19, 2007.

[8] M. Khatibi and F. Khormaei, "Learning and personality: A review,"J. Educ. Manag. Stud., vol. 6, no. 4, pp. 89-97, 2016.

[9] C. Okoli, "A Guide to Conducting a Standalone Systematic Literature Review,” Commun. Assoc. Inf. Syst., vol. 37, pp. 879-910, 2015.

[10] D. Moher et al., "Preferred reporting items for systematic review and and explanation meta-analysis protocols (PRISMA-P) 2015: elaboration and explanation,” Rev. Esp. Nutr. Humana y Diet., vol. 20, no. 2, pp. 148-160, 2015.

[11] K. Watkins, N. Forge, T. Lewinson, B. Garner, L. D. Carter, and L. Greenwald, "Undergraduate Social Work Students' Perceptions of a Team-Based Learning Approach to Exploring Adult Development,” J. Teach. Soc. Work, vol. 38, no. 2, pp. 214-234, 2018.

[12] S. Salimath, M. Vijaylakshmi, and A. S. Shettar, "A Comparative Study of Team Based Learning and Individual Learning," 2018.

[13] L. M. Jeno et al., "The relative effect of team-based learning on motivation and learning: A self-determination theory perspective," CBE Life Sci. Educ., vol. 16, no. 4, pp. 1-12, 2017.

[14] R. Rezaee, N. Moadeb, and N. Shokrpour, “Team-Based Learning: A 
New Approach Toward Improving Education,” no. 5, 2015.

[15] M. Nicole and L. Larson, “Team Learning Behaviors: Supporting Team-Based Learning in a First-Year Design and Communications Course," 2016.

[16] H. Kim, Y. Song, R. Lindquist, and H. Kang, "Effects of team-based learning on problem-solving , knowledge and clinical performance of Korean nursing students,” Nurse Educ. Today, vol. 38, pp. 115-118, 2016.

[17] P. L. Hettler, "Student Demographics and the Impact of Team-Based Learning,” Int. Adv. Econ. Res., vol. 21, no. 4, pp. 413-422, 2015.

[18] T. R. Frame, S. M. Cailor, R. J. Gryka, A. M. Chen, M. E. Kiersma, and L. Sheppard, "Student perceptions of team-based learning vs traditional lecture-based learning,” Am. J. Pharm. Educ., 2015.

[19] C. Iramaneerat and O. S. Ba, "Assessing the Outcomes of TeamBased Learning in Surgery,” vol. 100, pp. 24-32, 2017.

[20] D. M. Miller, K. Khalil, O. Iskaros, and J. A. Van Amburgh, "Professional and pre-professional pharmacy students' perceptions of team based learning (TBL) at a private research-intensive university,” Curr. Pharm. Teach. Learn., vol. 9, no. 4, pp. 666-670, 2017.

[21] J. Stepanova, “Team-Based Learning in Business English,” pp. 12- 13, 2017.

[22] A. S. Obad et al., “Assessment of first-year medical students , perceptions of teaching and learning through team-based learning sessions,” pp. 536-542, 2019.
[23] K. C. Behling, M. M. Murphy, and O. J. Lopez, "Team-Based Learning in a Pipeline Course in Medical Microbiology for UnderRepresented Student Populations in Medicine Improves Learning of Microbiology Concepts †,” J. Microbiol. Biol. Educ., vol. 17, no. 3, pp. 370-379, 2017.

[24] N. A. S. Ismail, "Effectiveness of Team-Based Learning in teaching Medical Genetics to Medical Undergraduates,” Malays J Med Sci, vol. 23, no. 2, pp. 73-77, 2016.

[25] T. R. Frame, R. Gryka, M. E. Kiersma, A. L. Todt, S. M. Cailor, and A. M. H. Chen, "Student Perceptions of and Confidence in Self-Care Course Concepts Using Team-based Learning,” vol. 80, no. 3, 2016.

[26] P. Kenny, H. Mclaren, M. Blissenden, and S. Villios, "Improving the Students Tax Experience: A Team-based Learning Approach for Undergraduate Accouting Students,” vol. 10, no. 1, pp. 43-65, 2015.

[27] M. Greetham and K. Ippolito, "Instilling collaborative and reflective practice in engineers: using a team-based learning strategy to prepare students for working in project teams,” High. Educ. Pedagog., vol. 3, no. 1, pp. 510-521, 2018.

[28] H. Topi and R. T. Wright, "Differentiating Information Systems and Information Technology as Fields of Study: An Evaluation of Model Curricula,” in Siged 2013, 2013, pp. 1-13. 